\title{
Stability of fractional neutral systems
}

\author{
KeWei Liu ${ }^{1,2^{*}}$ and Wei Jiang ${ }^{1}$
}

"Correspondence: kweiliu@163.com ${ }^{1}$ School of Mathematical Sciences, Anhui University, Hefei, 230039, China

${ }^{2}$ School of Mathematics, Hefei University of Technology, Hefei, 230009, China

\begin{abstract}
In this paper, we investigate the stability of a class of nonlinear fractional neutral systems. We extend the Lyapunov-Krasovskii approach to nonlinear fractional neutral systems. Necessary and sufficient conditions for stability are obtained for the nonlinear fractional neutral systems.
\end{abstract}

MSC: $34 \mathrm{~K} 20 ; 34 \mathrm{~K} 37 ; 34 \mathrm{~K} 40$

Keywords: fractional neutral systems; stability; Lyapunov-Krasovskii approach

\section{Introduction}

In the past few decades, fractional calculus and fractional differential equations have attracted great attention. It has been proved that fractional-order calculus is more adequate to describe real world problems than the integer calculus. Therefore, not only mathematicians have currently a strong interest in the fractional calculus but also researchers in applied fields such as mechanics, physics, chemistry, biology, economics, control theory, and signal processing. For details and examples, see [1-7] and the references therein.

On the other hand, stability analysis is always one of the most important issues in the theory of differential equations and their applications. The analysis on stability of fractionalorder differential equations is more complex than that of classical differential equations, since fractional derivatives are nonlocal and have weakly singular kernels. The earliest study on the stability of fractional systems started in [8]; the author has given a well-known stability criterion for a linear fractional differential system with constant coefficient matrix. Since then, the stability of fractional systems has attracted increasing interest. Many researchers have done further studies on the stability of fractional systems. In [9], by the frequency domain method, the BIBO-stability of fractional differential systems with delays was considered. Chen and Moore [10] considered the analytical stability bound for a class of fractional differential systems with time-delay. The authors derived a stability condition by applying the Laplace transformation and the Lambert function. [11] studied the linear system with multi-order Caputo derivative and derived a sufficient condition on Lyapunov global asymptotical stability. In [12] LMI stability conditions for linear fractional differential systems were given. The boundedness properties of system responses are very important from the engineering point of view. From this fact, finite-time stability for fractional differential systems with time-delay was introduced [13, 14]. In [15], the definitions of $q$-fractional calculus was presented and the stability of non-autonomous systems within the frame of the $q$-Caputo fractional derivative was studied. Recently, survey papers [16, 17] have provided more details about the stability results and the methods available to an-

○2014 Liu and Jiang; licensee Springer. This is an Open Access article distributed under the terms of the Creative Commons Attribution License (http://creativecommons.org/licenses/by/2.0), which permits unrestricted use, distribution, and reproduction in any medium, provided the original work is properly cited. 
alyze the stability of fractional differential systems; the reader may refer to them and the references therein.

As is well known, Lyapunov's second method provides a way to analyze the stability of a system without explicitly solving the differential equations. It is necessary to extend Lyapunov's second method to fractional systems. In [18, 19], the fractional Lyapunov's second method was proposed, and the authors extended the exponential stability of integerorder differential system to the Mittag-Leffler stability of fractional differential system. In [20], by using Bihari's and Bellman-Gronwall's inequality, an extension of Lyapunov's second method for fractional-order systems was proposed. In [21-23], Baleanu et al. extended Lyapunov's method to fractional functional differential systems and developed the Lyapunov-Krasovskii stability theorem, Lyapunov-Razumikhin stability theorem and Mittag-Leffler stability theorem for fractional functional differential systems. As far as we know, there are few papers with respect to the stability of fractional neutral systems. In this paper, we consider the stability of a class of nonlinear fractional neutral functional differential equations with the Caputo derivative. Motivated by Li et al.. [18, 19], Baleanu et al. [21], and Cruz and Hale [24], we aim in this paper to extend the Lyapunov-Krasovskii approach for the nonlinear fractional neutral systems.

The rest of the paper is organized as follows. In Section 2, we give some notations and recall some concepts and preparation results. In Sections 3, by using Lyapunov functionals, we extend the Lyapunov-Krasovskii approach for nonlinear fractional neutral systems, results of stability for nonlinear fractional neutral systems are presented. Finally, some concluding remarks end the paper.

\section{Preliminaries}

In this section, we introduce notations, definitions, and preliminary facts needed here. Throughout this paper, let $\mathbb{R}^{n}$ be a real $n$-dimensional linear vector space with the norm $|\cdot|,\|A\|_{0}$ denotes the induced norm of a matrix $A$, let $\mathcal{C}=C\left([-r, 0], \mathbb{R}^{n}\right)$ be the space of continuous functions taking $[-r, 0]$ into $\mathbb{R}^{n}$ with $\|\phi\|, \phi \in \mathcal{C}$ defined by $\|\phi\|=\sup _{s \in[-r, 0]}|\phi(s)|$, $\mathcal{C}(M)=\{\phi \in \mathcal{C}:\|\phi\| \leq M\}, r>0, M>0$ are constants. If $\sigma \in \mathbb{R}, A>0$ and $x \in C([\sigma-r, \sigma+$ $\left.A], \mathbb{R}^{n}\right)$, then for any $t \in[\sigma, \sigma+A]$, we let $x_{t} \in \mathcal{C}$ be defined by $x_{t}(\theta)=x(t+\theta), \theta \in[-r, 0]$.

Let us recall the following definitions. For more details, we refer the reader to $[1,3,4]$.

Definition 2.1 The fractional-order integral of a function $f:\left[t_{0}, \infty\right) \rightarrow \mathbb{R}$ of order $\alpha \in$ $\mathbb{R}^{+}=[0,+\infty)$ is defined by

$$
I_{t_{0}}^{\alpha} f(t)=\frac{1}{\Gamma(\alpha)} \int_{t_{0}}^{t}(t-s)^{\alpha-1} f(s) d s
$$

where $\Gamma(\cdot)$ is the gamma function.

Definition 2.2 For a function $f$ given on the interval $\left[t_{0}, \infty\right)$, the $\alpha$ order RiemannLiouville fractional derivative of $f$ is defined by

$$
D_{t_{0}}^{\alpha} f(t)=\frac{1}{\Gamma(n-\alpha)}\left(\frac{d}{d t}\right)^{n} \int_{t_{0}}^{t}(t-s)^{n-\alpha-1} f(s) d s, \quad n-1<\alpha<n, n \in \mathbb{N}
$$

where $\mathbb{N}=\{1,2,3, \ldots\}$. 
Definition 2.3 For a function $f$ given on the interval $\left[t_{0}, \infty\right)$, the $\alpha$ order Caputo fractional derivative of $f$ is defined by

$$
\begin{aligned}
& { }^{c} D_{t_{0}}^{\alpha} f(t)=\frac{1}{\Gamma(n-\alpha)} \int_{t_{0}}^{t}(t-s)^{n-\alpha-1} f^{(n)}(s) d s, \quad n-1<\alpha<n, \\
& { }^{c} D_{t_{0}}^{\alpha} f(t)=f^{(n)}(t), \quad \alpha=n, n \in \mathbb{N} .
\end{aligned}
$$

Some properties of the aforementioned operators are recalled below $[1,3,4]$.

Property 2.1 The following results are especially interesting:

(i) For $v>-1$, we have $D_{t_{0}}^{\alpha}\left(t-t_{0}\right)^{v}=\frac{\Gamma(1+v)}{\Gamma(1+v-\alpha)}\left(t-t_{0}\right)^{\nu-\alpha}$.

(ii) When $n-1<\alpha<n, n \in \mathbb{N}$, we have

$$
{ }^{c} D_{t_{0}}^{\alpha} f(t)=D_{t_{0}}^{\alpha}\left[f(t)-\sum_{i=0}^{n-1} \frac{f^{(i)}\left(t_{0}\right)\left(t-t_{0}\right)^{i}}{i !}\right] .
$$

(iii) For $\alpha \in(0,1), T \geq t_{0}$ and $f \in C\left(\left[t_{0}, T\right], \mathbb{R}^{n}\right)$, we have ${ }^{c} D_{t_{0}}^{\alpha} I_{t_{0}}^{\alpha} f(t)=f(t)$, $I_{t_{0}}^{\alpha c} D_{t_{0}}^{\alpha} f(t)=f(t)-f\left(t_{0}\right)$.

Remark 2.1 From Property 2.1, if ${ }^{c} D_{t_{0}}^{\alpha} f(t) \geq 0, \alpha \in(0,1)$, then for $t \geq t_{0}$, we have:

(i) $f(t) \geq f\left(t_{0}\right)$.

(ii) In general, it is not true that $f(t)$ is nondecreasing in $t$.

In [24], Cruz and Hale studied a class of functional difference operators which are very useful in stability theory and the asymptotic behavior of solutions of functional differential equations of neutral type. In a monograph [25], Hale presents the following definition and results of the difference operators.

Definition 2.4 Suppose $\mathcal{D}: \mathcal{C} \rightarrow \mathbb{R}^{n}$ is linear, continuous, and atomic at 0 and let $\mathcal{C}_{\mathcal{D}}=$ $\{\phi \in \mathcal{C}: \mathcal{D}(\phi)=0\}$. The operator $\mathcal{D}$ is said to be stable if the zero solution of the homogeneous 'difference' equation,

$$
\mathcal{D}\left(y_{t}\right)=0, \quad t \geq 0, y_{0}=\varphi \in \mathcal{C}_{\mathcal{D}},
$$

is uniformly asymptotically stable.

Lemma 2.1 The following statements are equivalent:

(1) $\mathcal{D}$ is stable.

(2) There are constants $a>0$ and $b>0$ such that for any $h \in C\left([0,+\infty), \mathbb{R}^{n}\right)$, any solution $y$ of the nonhomogeneous equation

$$
\mathcal{D}\left(y_{t}\right)=h(t), \quad t \geq 0, y_{0}=\varphi,
$$

satisfies

$$
\left\|y_{t}(0, \varphi)\right\| \leq b e^{-a t}\|\varphi\|+b \sup _{u \in[0, t]}|h(u)|, \quad t \geq 0 .
$$


Remark 2.2 Let $\mathcal{D}\left(x_{t}\right)=x(t)-B x(t-r)$, let the matrix $B$ be Schur stable, i.e., the spectrum of the matrix lies in the open unit disc of the complex plane; then $\mathcal{D}$ is stable.

\section{Stability criteria}

In this section, we consider the stability of the following nonlinear fractional neutral differential difference system:

$$
{ }^{c} D_{t_{0}}^{\alpha}\left[\mathcal{D}\left(x_{t}\right)\right]=f\left(t, x_{t}\right), \quad t>t_{0},
$$

with the initial condition

$$
x_{t_{0}}=\varphi,
$$

where $0<\alpha<1, \mathcal{D}\left(x_{t}\right)=x(t)-B x(t-r)$, the matrix $B$ is Schur stable, $f: \mathbb{R} \times \mathcal{C} \rightarrow \mathbb{R}^{n}$ is continuous, Lipschitz in $x_{t}$. Here, we always assume that fractional neutral system (3.1) with initial condition (3.2) has a unique continuous solution $x(t)$ which depends continuously upon $t_{0}, \varphi$.

If $V: \mathbb{R} \times \mathcal{C} \rightarrow \mathbb{R}$ is continuously differentiable, we define the Caputo fractional derivative ${ }^{c} D_{t_{0}}^{\gamma} V(t, \phi)$ along the solutions of (3.1)-(3.2) as

$$
{ }^{c} D_{t_{0}}^{\gamma} V\left(t, x_{t}\right)=\frac{1}{\Gamma(1-\gamma)} \int_{t_{0}}^{t}(t-s)^{-\gamma} V^{\prime}\left(s, x_{s}\right) d s
$$

Definition 3.1 We say that the zero solution $x=0$ of (3.1) is stable if for any $t_{0} \in \mathbb{R}$ and any $\varepsilon>0$, there exists a $\delta=\delta\left(t_{0}, \varepsilon\right)$ such that any solution $x(t)=x\left(t, t_{0}, \varphi\right)$ of (3.1) with initial value $\varphi$ at $t_{0},\|\varphi\|<\delta$ satisfies $|x(t)|<\varepsilon$ for $t \geq t_{0}$.

Theorem 3.1 Let $x=0$ be an equilibrium point of system (3.1). Then the zero solution of system (3.1) is stable if and only if there exist a functional $V: \mathbb{R} \times \mathcal{C}(M) \rightarrow \mathbb{R}$ and a continuous function $u(x)$ with $u(x)>0$ for $|x|>0$ and $u(0)=0$ such that the following conditions are satisfied:

(1) $V(t, 0)=0$.

(2) $V(t, \phi) \geq u(\mathcal{D}(\phi))$.

(3) For any given $t_{0}$ the functional $V\left(t_{0}, \phi\right)$ is continuous in $\phi$ at the point 0 , i.e., for any $\varepsilon>0$ there exists $\delta>0$ such that the inequality $\|\phi\|<\delta$ implies $\left|V\left(t_{0}, \phi\right)-V\left(t_{0}, 0\right)\right|=V\left(t_{0}, \phi\right)<\varepsilon$.

(4) Along the solutions of the system (3.1) the functional $V(t, \phi)$ satisfies $V\left(t, x_{t}\left(t_{0}, \varphi\right)\right) \leq V\left(t_{0}, \varphi\right)$ for $t \geq t_{0}$.

Proof Sufficiency: Since the matrix $B$ is Schur stable, there exist $L \geq 1$ and $0<\zeta<1$ such that the inequality $\left\|B^{k}\right\|_{0} \leq L \zeta^{k}$ holds for $k \geq 0$.

For a given $\varepsilon>0(\varepsilon<M)$, we first set $\varepsilon_{1}=\frac{\varepsilon(1-\zeta)}{L}>0$ and then introduce the positive value

$$
\eta\left(\varepsilon_{1}\right)=\min _{|x|=\varepsilon_{1}} u(x) .
$$

Since for a given $t_{0}$ functional $V\left(t_{0}, \phi\right)$ is continuous in $\phi$ at the point 0 , there exists $\delta_{1}\left(\varepsilon, t_{0}\right)$ such that $V\left(t_{0}, \phi\right)<\eta\left(\varepsilon_{1}\right)$ for any $\phi \in \mathcal{C}$, with $\|\phi\|<\delta_{1}\left(\varepsilon, t_{0}\right)$. Here, we claim that 
$\delta_{1}\left(\varepsilon, t_{0}\right) \leq \varepsilon_{1}$. Suppose this is not the case; then there exists an initial function $\varphi \in \mathcal{C}$ such that $\|\varphi\|<\delta_{1}\left(\varepsilon, t_{0}\right)$ and $|\mathcal{D}(\varphi)|=\varepsilon_{1}$. On the one hand, for this initial function we have $u(\mathcal{D}(\varphi)) \geq \eta\left(\varepsilon_{1}\right)$. On the other hand, $u(\mathcal{D}(\varphi)) \leq V\left(t_{0}, \varphi\right)<\eta\left(\varepsilon_{1}\right)$. The contradiction proves the desired inequality.

Now we take $\delta\left(\varepsilon, t_{0}\right)=\frac{\delta_{1}\left(\varepsilon, t_{0}\right)}{1+L \zeta}$. For $\varphi \in \mathcal{C}$ with $\|\varphi\|<\delta\left(\varepsilon, t_{0}\right)$, we have

$$
u\left(\mathcal{D}\left(x_{t}\left(t_{0}, \varphi\right)\right)\right) \leq V\left(t, x_{t}\left(t_{0}, \varphi\right)\right) \leq V\left(t_{0}, \varphi\right)<\eta\left(\varepsilon_{1}\right), \quad t \geq t_{0} .
$$

Next, we wish to show

$$
\left|\mathcal{D}\left(x_{t}\left(t_{0}, \varphi\right)\right)\right|<\varepsilon_{1}, \quad t \geq t_{0}
$$

Assume by contradiction that there exists a $t_{1} \geq t_{0}$ for which

$$
\left|\mathcal{D}\left(x_{t_{1}}\left(t_{0}, \varphi\right)\right)\right| \geq \varepsilon_{1}
$$

Since

$$
\left|\mathcal{D}\left(x_{t_{0}}\left(t_{0}, \varphi\right)\right)\right|=|\mathcal{D}(\varphi)| \leq(1+L \zeta)\|\varphi\|<\delta_{1}\left(\varepsilon, t_{0}\right) \leq \varepsilon_{1}
$$

and $\left|\mathcal{D}\left(x_{t}\left(t_{0}, \varphi\right)\right)\right|$ is a continuous function of $t$, there exists $\bar{t} \in\left[t_{0}, t_{1}\right]$ such that

$$
\left|\mathcal{D}\left(x_{\bar{t}}\left(t_{0}, \varphi\right)\right)\right|=\varepsilon_{1} .
$$

On the one hand, it follows from relation (3.4) that

$$
u\left(\mathcal{D}\left(x_{\bar{t}}\left(t_{0}, \varphi\right)\right)\right) \geq \eta\left(\varepsilon_{1}\right) .
$$

On the other hand, relation (3.5) provides the opposite inequality

$$
u\left(\mathcal{D}\left(x_{\bar{t}}\left(t_{0}, \varphi\right)\right)\right)<\eta\left(\varepsilon_{1}\right) .
$$

The contradiction proves that inequality (3.7) is wrong, and relation (3.6) is true. Then there exists a function $z(t)$ with $|z(t)|<\varepsilon_{1}, t \geq t_{0}$ such that

$$
x\left(t, t_{0}, \varphi\right)=B x\left(t-r, t_{0}, \varphi\right)+z(t), \quad t \geq t_{0} .
$$

For a given $t \geq t_{0}$, there must exist a positive integer number $k$ such that $t \in\left[t_{0}+(k-\right.$ 1) $r, t_{0}+k r$ ]. Iterating equality (3.8) $k-1$ times we obtain

$$
x\left(t, t_{0}, \varphi\right)=B^{k} x\left(t-k r, t_{0}, \varphi\right)+\sum_{i=0}^{k-1} B^{i} z(t-i r) .
$$

Since $t-k r \in\left[t_{0}-r, t_{0}\right]$,

$$
\left|x\left(t-k r, t_{0}, \varphi\right)\right| \leq\|\varphi\|<\delta\left(\varepsilon, t_{0}\right) \leq \varepsilon_{1},
$$


and we obtain the following inequality:

$$
\begin{aligned}
\left|x\left(t, t_{0}, \varphi\right)\right| & \leq\left\|B^{k}\right\|_{0}\left|x\left(t-k r, t_{0}, \varphi\right)\right|+\sum_{i=0}^{k-1}\left\|B^{i}\right\|_{0}|z(t-i r)| \\
& <L \zeta^{k} \delta\left(\varepsilon, t_{0}\right)+\sum_{i=0}^{k-1} L \zeta^{i} \varepsilon_{1}<\frac{L}{1-\zeta} \varepsilon_{1}=\varepsilon, \quad t \geq t_{0} .
\end{aligned}
$$

Therefore, the zero solution of system (3.1) is stable.

Necessity: Now, the zero solution of system (3.1) is stable, and we must prove that there exist a function $u(s)$ and a functional $V(t, \phi)$ that satisfy the conditions of the theorem.

Since the zero solution of system (3.1) is stable, for $\varepsilon=M$ there exists $\delta\left(M, t_{0}\right)$ such that the inequality $\|\varphi\|<\delta\left(M, t_{0}\right)$ implies that $\left|x\left(t, t_{0}, \varphi\right)\right|<M$ for $t \geq t_{0}$. We define the function $u(x)=|x|, x \in \mathbb{R}^{n}$ and the functional $V(t, \phi)$ as follows:

$$
V\left(t, x_{t}\left(t_{0}, \varphi\right)\right)= \begin{cases}\sup _{s \geq t}\left|\mathcal{D}\left(x_{s}\left(t_{0}, \varphi\right)\right)\right|, & \text { if }\left|x\left(s, t_{0}, \varphi\right)\right|<M, \text { for } s \geq t_{0}, \\ (1+L \zeta) M, & \text { if } \exists T \geq t_{0} \text { such that }\left|x\left(T, t_{0}, \varphi\right)\right| \geq M .\end{cases}
$$

Since for $\varphi=0$ the corresponding solution is trivial, $x\left(t, t_{0}, 0\right)=0, t \geq t_{0}$, we have $V(t, 0)=0$. In the case where $\left|x\left(s, t_{0}, \varphi\right)\right|<M$, for $s \geq t_{0}$, we have

$$
\begin{aligned}
u\left(\mathcal{D}\left(x_{t}\left(t_{0}, \varphi\right)\right)\right) & =\left|\mathcal{D}\left(x_{t}\left(t_{0}, \varphi\right)\right)\right| \\
& \leq \sup _{s \geq t}\left|\mathcal{D}\left(x_{s}\left(t_{0}, \varphi\right)\right)\right|=V\left(t, x_{t}\left(t_{0}, \varphi\right)\right) .
\end{aligned}
$$

In the other case where there exists $T \geq t_{0}$ such that $\left|x\left(T, t_{0}, \varphi\right)\right| \geq M$, the following inequality holds:

$$
u\left(\mathcal{D}\left(x_{t}\left(t_{0}, \varphi\right)\right)\right)=\left|\mathcal{D}\left(x_{t}\left(t_{0}, \varphi\right)\right)\right| \leq(1+L \zeta) M=V\left(t, x_{t}\left(t_{0}, \varphi\right)\right) .
$$

Further, for a given $t_{0}$, the stability of the zero solution means that for any $\varepsilon>0$ there exists $\delta_{1}=\delta\left(\frac{\varepsilon}{1+L \zeta}, t_{0}\right)$ such that $\|\varphi\| \leq \delta_{1}$ implies

$$
\left|x\left(t, t_{0}, \varphi\right)\right|<\frac{\varepsilon}{1+L \zeta}, \quad t \geq t_{0} .
$$

Then

$$
\left|\mathcal{D}\left(x_{t}\left(t_{0}, \varphi\right)\right)\right| \leq\left|x\left(t, t_{0}, \varphi\right)\right|+L \zeta\left|x\left(t-r, t_{0}, \varphi\right)\right|<\varepsilon, \quad t \geq t_{0} .
$$

Therefore,

$$
\left|V\left(t_{0}, \varphi\right)-V\left(t_{0}, 0\right)\right|=V\left(t_{0}, 0\right) \leq \varepsilon,
$$

that is, for a fixed $t_{0}$ the functional $V\left(t_{0}, \varphi\right)$ is continuous in $\varphi$ at the point 0 .

Finally, we need to show $V\left(t, x_{t}\left(t_{0}, \varphi\right)\right) \leq V\left(t_{0}, \varphi\right), t \geq t_{0}$. First, if $\left|x\left(s, t_{0}, \varphi\right)\right|<M$ for $s \geq t_{0}$,

$$
V\left(t_{0}, \varphi\right)=\sup _{s \geq t_{0}}\left|\mathcal{D}\left(x_{s}\left(t_{0}, \varphi\right)\right)\right|
$$


and

$$
V\left(t, x_{t}\left(t_{0}, \varphi\right)\right)=\sup _{s \geq t}\left|\mathcal{D}\left(x_{s}\left(t_{0}, \varphi\right)\right)\right|
$$

Note that $\{s: s \geq t\} \subseteq\left\{s: s \geq t_{0}\right\}$ for $t \geq t_{0}$; then we obtain

$$
V\left(t, x_{t}\left(t_{0}, \varphi\right)\right) \leq V\left(t_{0}, \varphi\right)
$$

In the second case, there exists $T \geq t_{0}$ such that $\left|x\left(T, t_{0}, \varphi\right)\right| \geq M$, we have

$$
V\left(t, x_{t}\left(t_{0}, \varphi\right)\right)=V\left(t_{0}, \varphi\right)=(1+L \zeta) M
$$

Therefore, we have

$$
V\left(t, x_{t}\left(t_{0}, \varphi\right)\right) \leq V\left(t_{0}, \varphi\right), \quad t \geq t_{0}
$$

The proof is complete.

Remark 3.1 The functional (3.9) has only academic value. Obviously, we cannot use such functionals in applications. The computation of practically useful Lyapunov functionals is a very difficult task.

Theorem 3.2 Let $x=0$ be an equilibrium point of system (3.1). Suppose $u(s)$ is a continuous function with $u(x)>0$ for $|x|>0$ and $u(0)=0$. If there exists a continuous functional $V$ : $\mathbb{R} \times \mathcal{C}(M) \rightarrow \mathbb{R}$ such that the following conditions are satisfied:

(1) $V(t, 0)=0$;

(2) $V(t, \phi) \geq u(\mathcal{D}(\phi))$;

(3) along the solutions of the system (3.1) the functional $V(t, \phi)$ is continuously differentiable and satisfies

$$
{ }^{c} D_{t_{0}}^{\beta} V\left(t, x_{t}\left(t_{0}, \varphi\right)\right) \leq 0, \quad t \geq t_{0}
$$

where $\beta \in(0,1]$,

then the zero solution of system (3.1) is stable.

Proof Note that the theorem's condition imply that of Theorem 3.1, therefore, the zero solution of system (3.1) is stable.

Theorem 3.3 Suppose that the assumptions in Theorem 3.2 are satisfied except for replac$\operatorname{ing}^{c} D_{t_{0}}^{\beta}$ by $D_{t_{0}}^{\beta}$; then one has the same result for stability.

Proof By using Property 2.1 we have

$$
{ }^{c} D_{t_{0}}^{\beta} V\left(t, x_{t}\right)=D_{t_{0}}^{\beta} V\left(t, x_{t}\right)-V\left(t_{0}, \varphi\right) \frac{\left(t-t_{0}\right)^{-\beta}}{\Gamma(1-\beta)} .
$$

Since $V\left(t_{0}, \varphi\right) \geq 0$, then ${ }^{c} D_{t_{0}}^{\beta} V\left(t, x_{t}\right) \leq D_{t_{0}}^{\beta} V\left(t, x_{t}\right)$. Then we obtain the same result for stability. 


\section{Conclusions}

In this paper, we have studied the stability of a class of nonlinear fractional neutral differential difference systems. We introduce the Lyapunov-Krasovskii approach for fractional neutral systems, which enrich the knowledge of both system theory and fractional calculus. By using Lyapunov functionals and the Lyapunov-Krasovskii technique, stability criteria are obtained for the nonlinear fractional neutral systems. Finally, we point out that since the computation of practically useful Lyapunov functionals is a very difficult task, the fractional Lyapunov method has its own limitations and should be generalized and verified for more complicated linear and nonlinear problems.

Competing interests

The authors declare that they have no competing interests.

\section{Authors' contributions}

All authors contributed equally in this article. They read and approved the final manuscript.

\section{Acknowledgements}

This work is supported by the National Natural Science Foundation of China (11371027), the Fundamental Research Funds for the Central Universities (2013HGXJ0226) and the Fund of Anhui University Graduate Academic Innovation Research (10117700004)

Received: 22 October 2013 Accepted: 19 February 2014 Published: 07 Mar 2014

\section{References}

1. Kilbas, AA, Srivastava, HM, Trujillo, JJ: Theory and Applications of Fractional Differential Equations. North-Holland Mathematics Studies, vol. 204. Elsevier, Amsterdam (2006)

2. Monje, CA, Chen, YQ, Vinagre, BM, Xue, D, Feliu, V: Fractional-Order Systems and Controls: Fundamentals and Applications. Springer, London (2010)

3. Podlubny, I: Fractional Differential Equations. Academic Press, San Diego (1999)

4. Samko, SG, Kilbas, AA, Marichev, Ol: Fractional Integrals and Derivatives: Theory and Applications. Gordon \& Breach, Yverdon (1993)

5. Biagini, F, Hu, Y, Øksendal, B, Zhang, B: Stochastic Calculus for Fractional Brownian Motion and Applications. Springer, London (2008)

6. Agrawal, RP: Certain fractional q-integrals and q-derivatives. Proc. Camb. Philos. Soc. 66, 365-370 (1969)

7. Sakthivel, R, Suganya, S, Anthoni, SM: Approximate controllability of fractional stochastic evolution equations. Comput. Math. Appl. 63, 660-668 (2012)

8. Matignon, D: Stability results for fractional differential equations with applications to control processing. In: IMACS-SMC Proceedings, Lille, France (1996)

9. Bonnet, C, Partington, JR: Analysis of fractional delay systems of retarded and neutral type. Automatica 38, 1133-1138 (2002)

10. Chen, YQ, Moore, $\mathrm{KL}$ : Analytical stability bound for a class of delayed fractional-order dynamic systems. Nonlinear Dyn. 29, 191-200 (2002)

11. Deng, $\mathrm{WH}, \mathrm{Li}, \mathrm{CP}, \mathrm{Lü}, \mathrm{JH}$ : Stability analysis of linear fractional differential system with multiple time delays. Nonlinear Dyn. 48(4), 409-416 (2007)

12. Sabatier, J, Moze, M, Farges, C: LMI stability conditions for fractional order systems. Comput. Math. Appl. 59, 1594-1609 (2010)

13. Lazarevic, MP, Spasic, AM: Finite-time stability analysis of fractional order time-delay systems: Gronwall's approach. Math. Comput. Model. 49, 475-481 (2009)

14. Liu, KW, Jiang, W: Finite-time stability of linear fractional order neutral systems. Math. Appl. 24(4), 724-730 (2011)

15. Jarad, F, Abdeljawad, T, Baleanu, D: Stability of q-fractional non-autonomous systems. Nonlinear Anal., Real World Appl. 14, 780-784 (2013)

16. Li, CP, Zhang, FR: A survey on the stability of fractional differential equations. Eur. Phys. J. Spec. Top. 193, 27-47 (2011)

17. Rivero, M, Rogosin, SV, Machado, JAT, Trujillo, JJ: Stability of fractional order systems. Math. Probl. Eng. 2013, Article ID $356215(2013)$

18. Li, Y, Chen, YQ, Podlubny, I: Mittag-Leffler stability of fractional order nonlinear dynamic systems. Automatica 45(8), 1965-1969 (2009)

19. Li, Y, Chen, YQ, Podlubny, I: Stability of fractional-order nonlinear dynamic systems: Lyapunov direct method and generalized Mittag-Leffler stability. Comput. Math. Appl. 59, 1810-1821 (2010)

20. Delavari, H, Baleanu, D, Sadati, J: Stability analysis of Caputo fractional-order nonlinear systems revisited. Nonlinear Dyn. 67(4), 2433-2439 (2012)

21. Baleanu, D, Ranjbar, A, Delavari, H, Sadati, JR, Abdeljawad, T, Gejji, V: Lyapunov-Krasovskii stability theorem for fractional systems with delay. Rom. J. Phys. 56, 636-643 (2011)

22. Baleanu, D, Sadati, SJ, Ghaderi, R, Ranjbar, A, Abdeljawad, T, Jarad, F: Razumikhin stability theorem for fractional systems with delay. Abstr. Appl. Anal. 2010, Article ID 124812 (2010)

23. Sadati, SJ, Baleanu, D, Ranjbar, A, Ghaderi, R, Abdeljawad, T: Mittag-Leffler stability theorem for fractional nonlinear systems with delay. Abstr. Appl. Anal. 2010, Article ID 108651 (2010)

24. Cruz, MA, Hale, JK: Stability of functional differential equations of neutral type. J. Differ. Equ. 7, 334-355 (1970)

25. Hale, JK: Theory of Functional Differential Equations. Springer, New York (1977) 
10.1186/1687-1847-2014-78

Cite this article as: Liu and Jiang: Stability of fractional neutral systems. Advances in Difference Equations 2014, 2014:78

Submit your manuscript to a SpringerOpen ${ }^{\circ}$ journal and benefit from:

- Convenient online submission

- Rigorous peer review

- Immediate publication on acceptance

Open access: articles freely available online

- High visibility within the field

- Retaining the copyright to your article

Submit your next manuscript at $\boldsymbol{s p r i n g e r o p e n . c o m ~}$ 